\title{
The compact Green's function for multiple bodies
}

\author{
P. J. Baddoo* and L. J. Ayton ${ }^{\dagger}$ \\ Department of Applied Mathematics and Theoretical Physics, Centre for Mathematical Sciences, Wilberforce Road, \\ Cambridge, CB3 OWA, UK
}

\begin{abstract}
The acoustic emissions of compact bodies may be analysed using the compact Green's function. Whilst it is straightforward to construct the compact Green's function for single bodies, the construction for multiple bodies is far more challenging. In this paper, we present analytic solutions for the compact Green's function for multiple bodies. By writing the solution in terms of the transcendental Schottky-Klein prime function, the compact Green's function has exactly the same functional form for any number of bodies in the system. Moreover, the formulation permits the inclusion of a low Mach number background flow. The compact Green's function is illustrated for some simple examples for flat plates and cylinders and the sound generation of a Kasper wing is considered in detail.
\end{abstract}

\section{Introduction}

The scattering of sound by objects remains a canonical problem in aeroacoustics. Significant effort has been expended in the study of single bodies whereas systems involving multiple bodies have received considerably less attention. The acoustic interactions of multiple bodies is nevertheless a topic of significance in a range of fields. In nature fliers and swimmers often operate in formation or close to a wall in order to enhance their propulsive efficiency. Moreover, multi-body interactions are ubiquitous in practical engineering applications in the form of high-lift aerofoils, turbomachinery, and take-off and landing scenarios. In this article we present analytical solutions for the sound generated by multiple interacting bodies. An elegant feature of the solution is that it is valid for any number of objects and any geometry.

The compact Green's function [1] is a powerful tool for modelling the production of sound. By assuming that the length scale of the scattering objects is small in comparison to the acoustic wavelength, the Green's function can be written in terms of the so-called Kirchhoff vectors. These quantities essentially represent a potential flow around the body and are therefore amenable to methods of complex variable theory. However, the Kirchhoff vectors are difficult to calculate for multiple bodies, and numerical techniques often must be employed [2-4]. In this article we derive analytic expressions for the Kirchhoff vectors, thus rendering vortex panel techniques largely unnecessary for this type of problem.

These analytic expressions are made possible by recent advances in complex variable theory. The existence of the Schottky-Klein prime function (henceforth referred to as the "prime function") has been known since 1897 where it was recorded in a monograph by Baker [5]. However, its fundamental significance as a building block in the study of multiply connected domains has not been recognised until relatively recently [6-8]. Moreover, the link between the prime function and the study of potential flows has only been realised in the last 15 years [9--11]. This research is particularly timely because the practical computation of the prime function has only been made possible through the recent development of a rapid numerical algorithm [12]. In this paper we demonstrate that these advances may be leveraged to the theory of multi-body sound generation. By taking advantage of various identities for the prime function, we are able to derive concise expressions for the Kirchhoff vectors that are valid for any number of objects.

The structure of the paper is as follows: in \$II we recall the form of the compact Green's function. In \$III we present the Schottky-Klein prime function and derive the analytic expressions for the Kirchhoff vectors. We plot the Kirchhoff vectors and compact Green's function for simple geometries such as flat plates and cylinders. In \$[V] we extend the result to consider objects that are in a background flow and consider the example of a Kasper wing. Finally, in $8 \sqrt{\mathrm{V}}$ we provide some conclusions and suggestions for future work. 


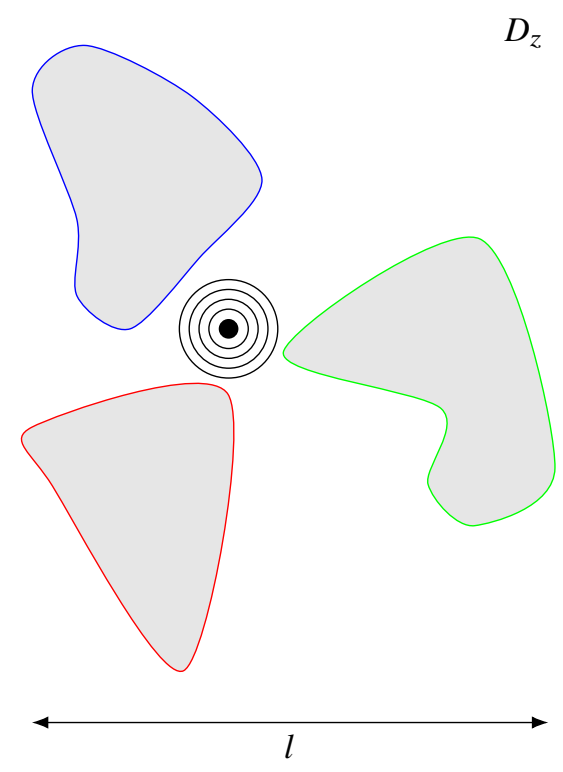

Fig. 1 Schematic diagram of the arrangement: an acoustic source is close to a system of bodies with characteristic lengthscale $l$. In this case there are 3 bodies, i.e. $M=2$. This domain is the $z$-plane.

\section{The compact Green's function}

We consider an arrangement of $M+1$ bodies where the total system has characteristic length scale $l$, as illustrated in figure 1. The entire system is labelled $S$ and the $m$-th body has boundary $\partial D_{m}$. For a more detailed derivation of the compact Green's function, the reader is referred to [1]. The compact Green's function $\hat{G}(\boldsymbol{x}, \boldsymbol{y}, \omega)$ satisfies

$$
\left(\nabla^{2}+\kappa_{0}^{2}\right) \hat{G}(\boldsymbol{x}, \boldsymbol{y}, \omega)=\delta(\boldsymbol{x}-\boldsymbol{y}) .
$$

The compact assumption ensures that $\kappa_{0} l \ll 1$. By applying the reciprocity property we may interchange the positions of observer and source. Now the source is at the far-field point $\boldsymbol{x}$ and the observer is close to the bodies at $\boldsymbol{y}$ so that $|\boldsymbol{y}| \sim l$. Consequently, the compact Green's function satisfies

$$
\left(\frac{\partial^{2}}{\partial y_{1}^{2}}+\frac{\partial^{2}}{\partial y_{2}^{2}}+\frac{\partial^{2}}{\partial y_{3}^{2}}+\kappa_{0}^{2}\right) \hat{G}(\boldsymbol{y}, \boldsymbol{x}, \omega)=\delta(\boldsymbol{y}-\boldsymbol{x}),
$$

subject to the no-flux boundary condition on each object

$$
\frac{\partial \hat{G}}{\partial x_{n}}=0 \quad \text { for } x \in \partial D_{m} \text { for } m=0 \ldots M-1 .
$$

The symmetric form of the compact Green's function may be derived as [13]

$$
\hat{G}(\boldsymbol{y}, \boldsymbol{x}, \omega)=-\frac{\mathrm{e}^{\mathrm{i}|\mathbf{X}-\mathbf{Y}|}}{4 \pi|\mathbf{X}-\mathbf{Y}|} \quad \text { as }|\boldsymbol{x}| \rightarrow \infty,
$$

where $\mathbf{X}$ and $\mathbf{Y}$ are known as the Kirchhoff vectors for the observer and source respectively. We recall from [1] that the Kirchhoff vectors are defined such that

$$
\begin{aligned}
\nabla^{2} Y_{j} & =0 & & \forall \boldsymbol{y} \notin S, \\
\frac{\partial Y_{j}}{\partial y_{n}} & =0 & & \text { for } \boldsymbol{y} \in \partial D_{m} \text { for } m=0, \cdots, M, \\
Y_{j} & \sim y_{j} & & \text { as }|\boldsymbol{y}| \rightarrow \infty,
\end{aligned}
$$

*PhD Candidate, AIAA Student Member, baddoo@ damtp.cam.ac.uk

${ }^{\dagger}$ EPSRC Early Career Fellow, Churchill College, University of Cambridge, 1.j.ayton@ damtp.cam.ac.uk 


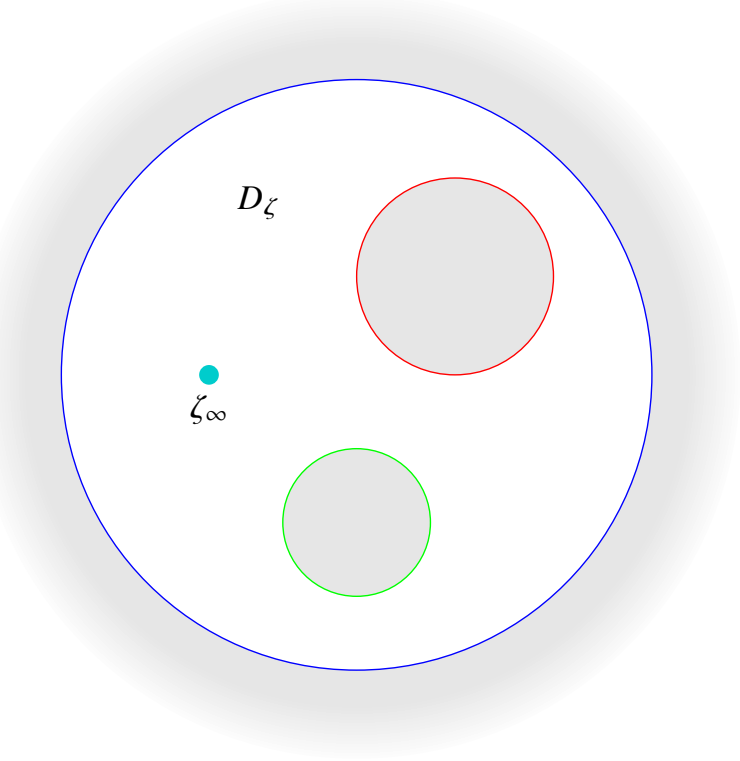

(a)
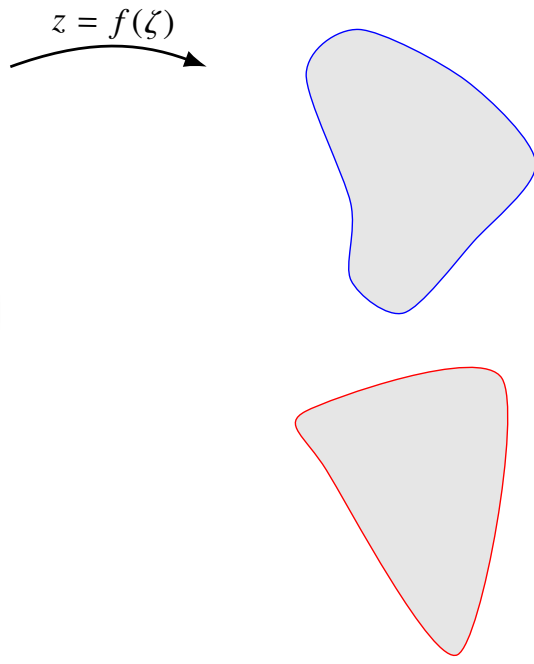

(b)

Fig. 2 a) The canonical circular domain made up of the unit disc with $M=2$ excised circles. b) The physical domain made up of $M=2$ objects. The conformal mapping between the two domains is indicated by the arrow. The unit disc is mapped to the blue object, the red disc is mapped to the red object, and the green disc is mapped to the green object. The point $\zeta_{\infty}$ is mapped to infinity.

which corresponds to the velocity potential of a uniform flow satisfying the no-flux condition. The uniqueness of $Y_{1,2}$ is closed by enforcing the circulation around every object to be zero. This condition can be derived from applying the causality principal in concert with Kelvin's circulation theorem [13]. Consequently, calculation of the compact Green's function reduces to the calculation of the uniform potential flow through the arrangement $S$ with zero circulation around every object.

\section{Analytic solutions for the Kirchhoff vectors}

In this section we derive analytic expressions for the Kirchhoff vectors $Y_{1,2}$. We calculate the Kirchhoff vectors in a canonical circular domain and then conformally map this circular domain to the physical domain of interest as illustrated in figure 2 ,

The canonical circular domain is labelled as $D_{\zeta}$. We define $D_{\zeta}$ to be the unit disc with $M$ excised discs so that there are $M+1$ boundary circles in total. We label the unit disc $|\zeta|=1$ as $C_{0}$, and the excised discs as $\left\{C_{j} \mid j=1, \cdots, M\right\}$. The excised discs have centers $\left\{q_{j} \mid j=1, \cdots, M\right\}$ and radii $\left\{\delta_{j} \mid j=1, \cdots, M\right\}$. A typical circular domain for $M=2$ is illustrated in figure 3 .

\section{A. Conformal mapping}

We label the conformal mapping between $D_{\zeta}$ and $D_{z}$ as $f$ so that

$$
z=f(\zeta)
$$

In general, the construction of $f$ has been a limiting factor in this area of research but there are now powerful tools available via the multiply connected Schwarz-Christoffel formula [8, 14, 16].

Note that the interior of the circular domain is mapped to the exterior of the physical arrangement in figure 2 . Consequently, there is a point, $\zeta=\zeta_{\infty}$ that is mapped to $z=\infty$ in the physical plane. As a result, close to $\zeta=\zeta_{\infty}$, the 


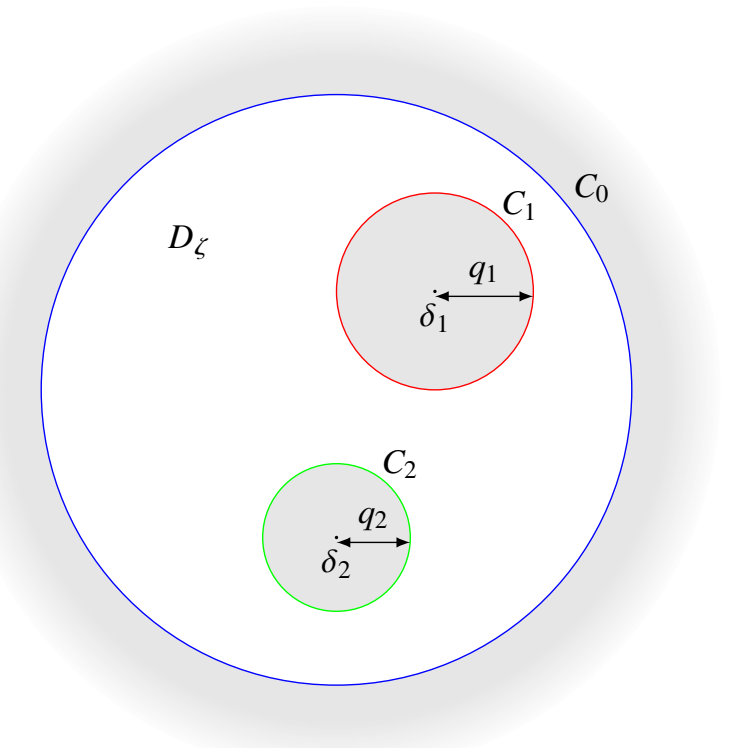

Fig. 3 A multiply connected circular domain $D_{\zeta}$ for $M=2$. $C_{0}$ denotes the unit circle and $\left\{C_{j} \mid j=1,2\right\}$ denote the excised discs with centers $\delta_{j}$ and radii $q_{j}$. The grey colour denotes regions that are outside the domain of definition.

conformal mapping will have the form

$$
z=f(\zeta) \sim \frac{a}{\zeta-\zeta_{\infty}}+\text { locally analytic function. }
$$

This expression will be necessary in calculating the Kirchhoff vectors. However, before we can proceed we must introduce a fundamental function in the study of multiply connected domains.

\section{B. The Schottky-Klein prime function}

In order to calculate the Kirchhoff vectors we must employ the Schottky-Klein prime function. The prime function was first discovered in 1897 [5] but has recently found prominence in the study of multiply connected domains such as those considered in the present study [6, 10]. Recent research [7] has shown that the prime function is a fundamental object in the analysis of multiply connected domains and is particularly applicable to the study of fluid problems involving multiple objects. Consequently, the prime function is a useful tool for constructing the Kirchhoff vectors.

The prime function is a transcendental analytic function associated with a particular canonical circular domain, such as that illustrated in 3 . For brevity, we suppress the $q_{j}$ and $\delta_{j}$ dependence of the prime function and write it as a bivariate function $\omega(\zeta, \alpha)$.

The general definition of the prime function is

$$
\omega(\zeta, \alpha)=(\zeta-\alpha) \prod_{\theta \in \Theta^{\prime \prime}} \frac{(\zeta-\theta(\alpha))(\alpha-\theta(\zeta))}{(\zeta-\theta(\zeta))(\alpha-\theta(\alpha))}
$$

where $\Theta^{\prime \prime}$ represents the Schottky group (which is the collection of all Möbius maps representing the reflections of excised circles in the unit disc) excluding the identity and all inverses [7]. This product formula may appear unwieldy, but in certain situations the infinite product may be written in more concise forms. For example, in the case $M=0$ the canonical circular domain is simply the unit disc and the prime function is therefore

$$
\omega(\zeta, \alpha)=\zeta-\alpha .
$$

When $M=1$, the canonical circular domain is the annulus

$$
\zeta=r \mathrm{e}^{\mathrm{i} \theta}, \quad q<r<1,0<\theta<2 \pi .
$$


In this case, the infinite product (5) may be written as

$$
\omega(\zeta, \alpha)=-\frac{\alpha}{C^{2}} P(\zeta / \alpha, q),
$$

where

$$
P(\zeta, q) \equiv(1-\zeta) \prod_{k=1}^{\infty}\left(1-q^{2 k} \zeta\right)\left(1-q^{2 k} \zeta^{-1}\right),
$$

and $C$ is a constant that will vanish in the subsequent analysis.

There are multiple tools available to compute the prime function numerically, as outlined in [12]. In this paper we use the recently developed numerical algorithm presented in [17] which is implemented in the SKPrime toolbox available in MATLAB (https://github.com/ACCA-Imperial/SKPrime).

\section{Construction of the Kirchhoff vectors}

The Kirchhoff vectors may now be calculated by using the new calculus of vortex dynamics as introduced in [10]. A fundamental function in this calculus is the hydrodynamic Green's function:

$$
W_{0}(\zeta, \alpha)=\frac{1}{2 \pi \mathrm{i}} \log \left(\frac{\omega(\zeta, \alpha)}{|\alpha| \omega(\zeta, 1 / \bar{\alpha})}\right) .
$$

The function $W_{0}$ has a $\log$ singularity at $\zeta=\alpha$ and has constant imaginary part on every circle. Consequently, it represents the complex potential of a point vortex at $\alpha$. It should be noted that the above expression is identical for any number of objects.

Crowdy [10] showed that the complex potential for a uniform flow through an arbitrary number of objects may be calculated using the hydrodynamic Green's function. The complex potential for uniform flow with speed $U$ in a direction making angle $\chi$ with the positive real axis is given by

$$
W_{U, \chi}(\zeta)=2 \pi U a \mathrm{i}\left[\mathrm{e}^{\mathrm{i} \chi} \frac{\partial W_{0}}{\partial \bar{\alpha}}-\mathrm{e}^{-\mathrm{i} \chi} \frac{\partial W_{0}}{\partial \alpha}\right]_{\alpha=\zeta_{\infty}},
$$

where $a$ is defined in (4).

We may compute the derivatives and write

$$
W_{U, \chi}(\zeta)=U a\left[\mathrm{e}^{\mathrm{i} \chi} \frac{\omega^{\prime}(\zeta, \alpha)}{\omega(\zeta, \alpha)}-\frac{\mathrm{e}^{-\mathrm{i} \chi}}{\bar{\alpha}^{2}} \frac{\omega^{\prime}(\zeta, 1 / \bar{\alpha})}{\omega(\zeta, 1 / \bar{\alpha})}\right]_{\alpha=\zeta_{\infty}} .
$$

It is now straightforward to calculate the Kirchhoff vectors in the horizontal and vertical directions by setting $\chi=0$ and $\pi / 2$ respectively to obtain the relevant complex potentials as

$$
\begin{aligned}
& W_{U, 0}(\zeta)=U a\left[\frac{\omega^{\prime}(\zeta, \alpha)}{\omega(\zeta, \alpha)}-\frac{1}{\bar{\alpha}^{2}} \frac{\omega^{\prime}(\zeta, 1 / \bar{\alpha})}{\omega(\zeta, 1 / \bar{\alpha})}\right]_{\alpha=\zeta_{\infty}}, \\
& W_{U, 0}(\zeta)=\mathrm{i} U a\left[\frac{\omega^{\prime}(\zeta, \alpha)}{\omega(\zeta, \alpha)}+\frac{1}{\bar{\alpha}^{2}} \frac{\omega^{\prime}(\zeta, 1 / \bar{\alpha})}{\omega(\zeta, 1 / \bar{\alpha})}\right]_{\alpha=\zeta_{\infty}} .
\end{aligned}
$$

The real parts of these quantities represent the velocity potential for flow that is uniform far from $S$. Moreover, they posses zero circulation around each body and therefore they are the unique solutions to the Kirchhoff vectors $Y_{1,2}$ and we may write

$$
\begin{aligned}
& Y_{1}(\zeta)=\mathbb{R e}\left[a\left[\frac{\omega^{\prime}(\zeta, \alpha)}{\omega(\zeta, \alpha)}-\frac{1}{\bar{\alpha}^{2}} \frac{\omega^{\prime}(\zeta, 1 / \bar{\alpha})}{\omega(\zeta, 1 / \bar{\alpha})}\right]_{\alpha=\zeta_{\infty}}\right], \\
& Y_{2}(\zeta)=\mathbb{R e}\left[\mathrm{i} a\left[\frac{\omega^{\prime}(\zeta, \alpha)}{\omega(\zeta, \alpha)}+\frac{1}{\bar{\alpha}^{2}} \frac{\omega^{\prime}(\zeta, 1 / \bar{\alpha})}{\omega(\zeta, 1 / \bar{\alpha})}\right]_{\alpha=\zeta_{\infty}}\right],
\end{aligned}
$$

where we have set $U=1$. Now that we have analytic forms for the Kirchhoff vectors, we can substitute the formulation into the expression for the compact Green's function (3). The compact Green's function for an acoustic source close to the arrangement for cylinders and flat plates is illustrated in figure 5 . We note that the expression (3) is only valid in the limit where the observer is in the far-field, i.e. $|x| \rightarrow \infty$. Nevertheless, the plots in figure 5 are an important illustrative tool for visualising the effects of multiple bodies on the production of sound. 


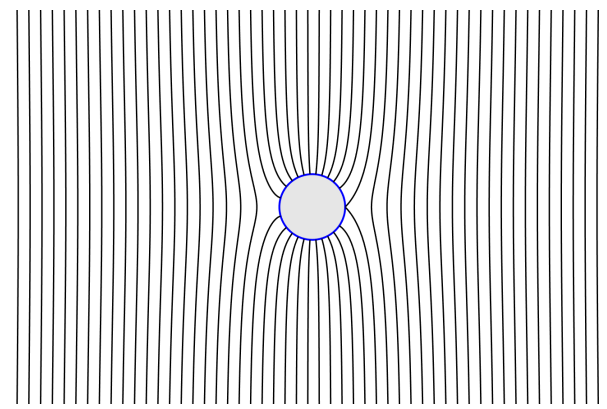

(a) $M=0$, Kirchhoff vector $Y_{1}$.

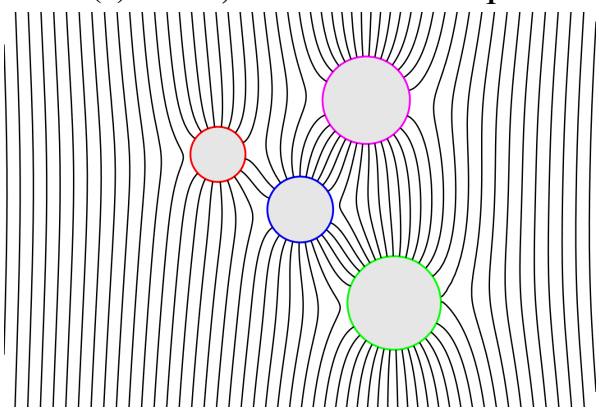

(c) $M=3$, Kirchhoff vector $Y_{1}$.

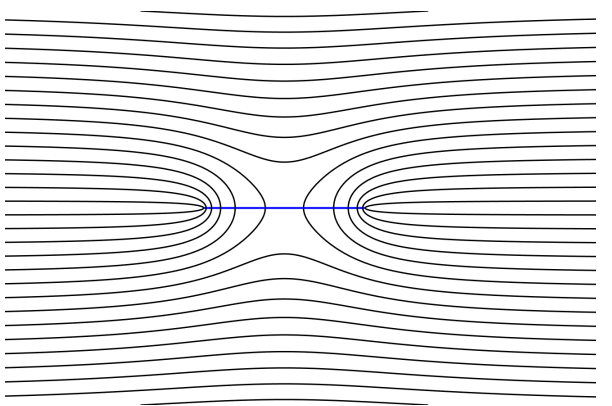

(b) $M=0$, Kirchhoff vector $Y_{2}$.

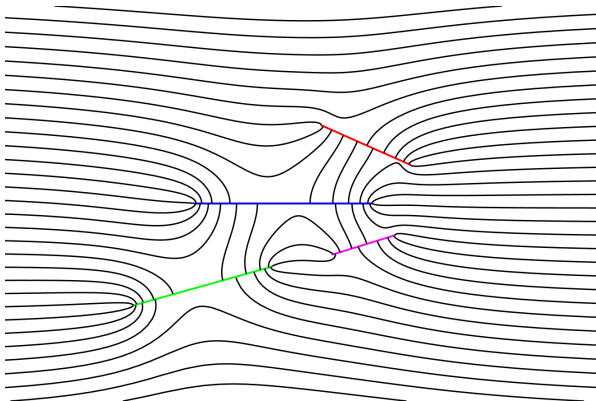

(d) $M=3$, Kirchhoff vector $Y_{2}$.

Fig. 4 The Kirchhoff vectors for an arrangement of objects. The figures on the left hand side show the Kirchhoff vector $Y_{1}$ for an array of cylinders, whilst the figures on the right hand side show the Kirchhoff vector $Y_{2}$ for an array of flat plates. The circulations around every object are zero. Note that these solutions are exact.

\section{Effects of background flow}

In many aeroacoustics applications the arrangement of objects is not stationary, but is travelling with some velocity. In this section we follow the approach of Howe [13, §1.11.3] to extend the compact Green's function to be applicable to problems involving low Mach number flows.

We assume that the arrangement of objects is in an irrotational, low Mach number flow $U_{j}(y)$ that is uniform far away from $S$. Consequently, the flow in the $j$ direction may be written in terms of the Kirchhoff vectors as

$$
U_{j}(\boldsymbol{y})=\mathbf{U}_{0} \cdot \nabla Y_{i}(\boldsymbol{y}),
$$

where $\mathbf{U}_{0}$ is the uniform mean velocity at infinity. We note that the Kirchhoff vectors (for any number of objects) have already been calculated in this article, so there is no further complexity involved in introducing background flow into the problem. This is one of the advantages of formulating the problem in terms of the Schottky-Klein prime function: both the compact Green's function and the background flow are derived from the same equations [11, 12]. The streamlines for the potential flow through arrangements of cylinders and flat plates are illustrated in figure 6 These examples have zero circulation around every body. The steady Kutta condition may be satisfied by employing the calculus of vortex dynamics [10] and placing vortices at the preimages of infinity with appropriate strengths. This allows the circulation to be specified around every object, which can be tuned to satisfy the Kutta condition.

The compact Green's function now satisfies the convected Helmholtz equation [13, (1.11.14)]

$$
\left(\frac{\partial^{2}}{\partial y_{j}^{2}}+\kappa_{0}^{2}-2 \mathrm{i} \kappa_{0} M_{j} \frac{\partial}{\partial y_{j}}\right) \hat{G}=\delta(\boldsymbol{x}-\boldsymbol{y}),
$$

where

$$
\mathbf{M}(\boldsymbol{y})=\frac{\mathbf{U}(\boldsymbol{y})}{c_{0}}, \quad \text { i.e. } \quad M_{j}=\frac{U_{j}(\boldsymbol{y})}{c_{0}} .
$$

Note that we have assumed that the Mach number is sufficiently small that $\mathcal{O}\left(M^{2}\right)$ terms can be ignored. 


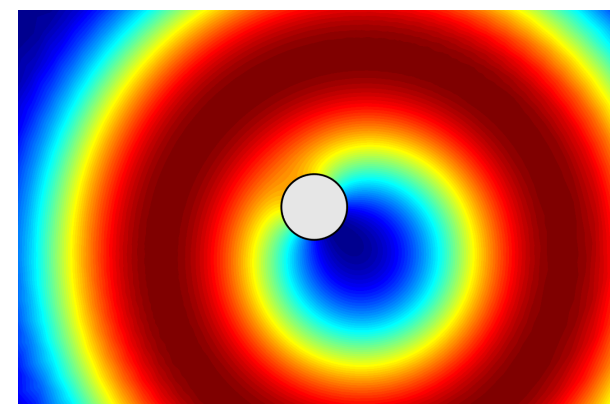

(a) $M=0$, compact Green's function $\hat{G}$.

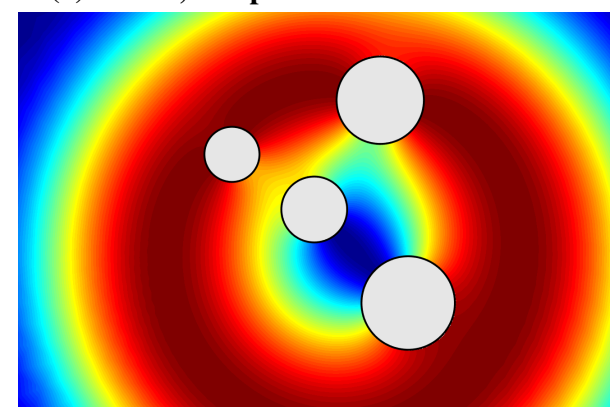

(c) $M=3$, compact Green's function $\hat{G}$.

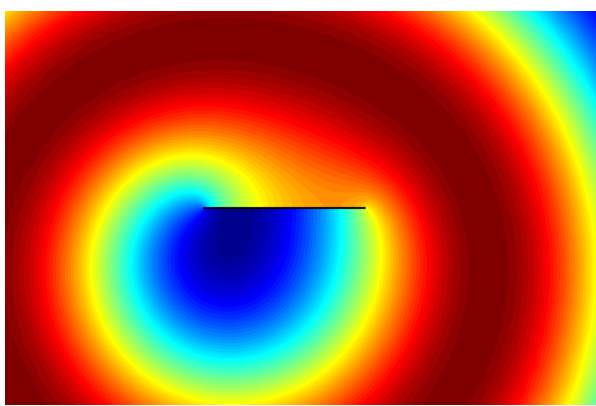

(b) $M=0$, compact Green's function $\hat{G}$.

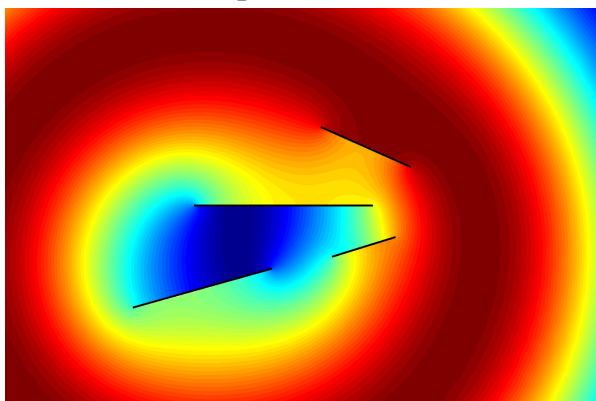

(d) $M=3$, compact Green's function $\hat{G}$.

Fig. 5 The compact Green's function vectors for some arrangements of objects. The figures on the left hand side show the Kirchhoff vector $Y_{1}$, whilst the figures on the right hand side show the Kirchhoff vector $Y_{2}$. The circulation around each cylinder is zero. In these plots the Green's function is normalised by $1 / r$ where $r$ is the distance between the observer and the source.

We now seek to transform the convected Helmholtz equation (13) to an equation amenable to previous methods. Taylor [18] showed that the governing equation for the Green's function in a steady homentropic potential flow at low Mach number can be reduced to the the typical Helmholtz equation by employing the transformation

$$
\hat{G}(\boldsymbol{x}, \boldsymbol{y} ; \omega)=\tilde{G}(\boldsymbol{x}, \boldsymbol{y} ; \omega) \mathrm{e}^{\mathrm{i} \kappa_{0} \mathbf{M}_{0} \cdot \mathbf{Y}} .
$$

Accordingly, the convected Helmholtz equation (13) becomes

$$
\left(\frac{\partial^{2}}{\partial y_{j}^{2}}+\kappa_{0}^{2}\right) \tilde{G}=\delta(\boldsymbol{x}-\boldsymbol{y}) \mathrm{e}^{-\mathrm{i} \kappa_{0} \mathbf{M}_{0} \cdot \mathbf{x}}
$$

and the solution for the transformed Green's function $\tilde{G}$ is

$$
\tilde{G}(\boldsymbol{x}, \boldsymbol{y} ; \omega)=-\frac{\exp \left[\mathrm{i} \kappa_{0}\left(|\mathbf{X}-\mathbf{Y}|-\mathbf{M}_{0} \cdot \mathbf{X}\right)\right]}{4 \pi|\mathbf{X}-\mathbf{Y}|} .
$$

Finally, the compact Green's function for a background flow with low Mach number is

$$
\hat{G}(\boldsymbol{x}, \boldsymbol{y} ; \omega)=-\frac{\exp \left[\mathrm{i} \kappa_{0}\left(|\mathbf{X}-\mathbf{Y}|-\mathbf{M}_{0} \cdot(\mathbf{X}-\mathbf{Y})\right)\right]}{4 \pi|\mathbf{X}-\mathbf{Y}|}
$$

The compact Green's function for sound production in low Mach number flows is illustrated for flat plates and cylinders in figure 7 .

\section{A. Example: the Kasper wing}

We now apply this new theory to a multi-element aerofoil. Kasper wings were first proposed [19] as a method of improving lift generation at moderate angles of attack by trapping vortices close to the wing. The wing features two tails at the trailing edge, as illustrated in figure 8 . 


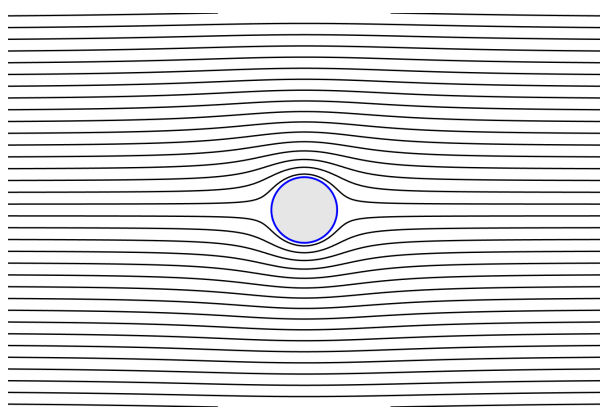

(a) $M=0$, horizontal flow.

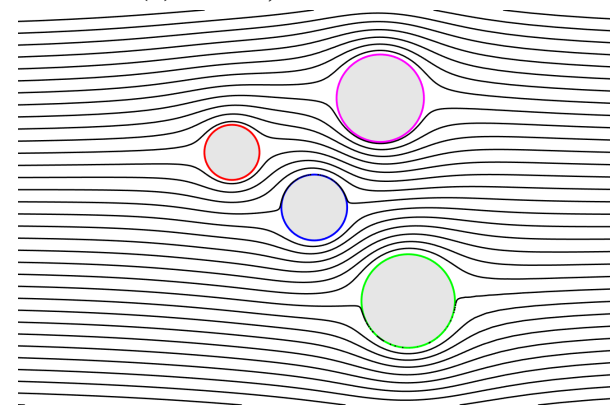

(c) $M=3$, horizontal flow.

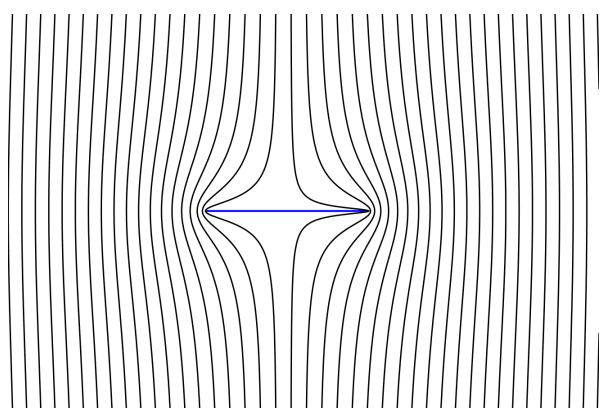

(b) $M=0$, vertical flow.

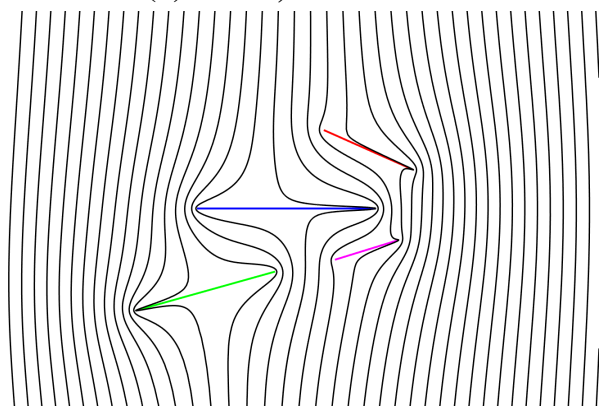

(d) $M=3$, vertical flow.

Fig. 6 The streamlines for potential flow past a series of objects. The figures on the left hand side show the streamlines for cylinders in horizontal flow whilst the figures on the right hand side show the streamlines for flat plates in vertical flow. Note that these solutions are exact.

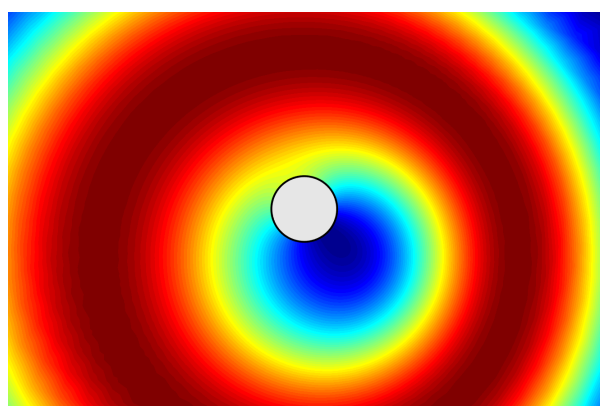

(a) $M=\mathbf{0}$, horizontal flow.

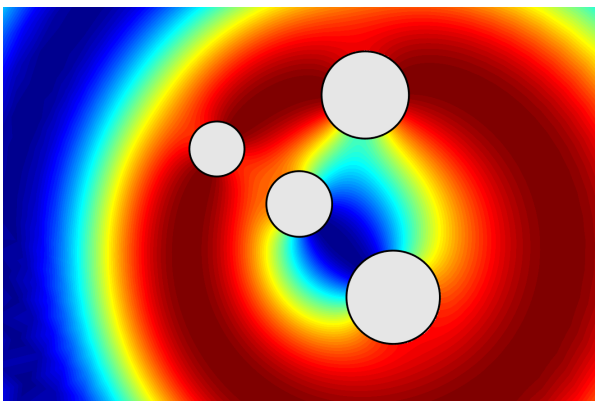

(c) $M=3$, horizontal flow.

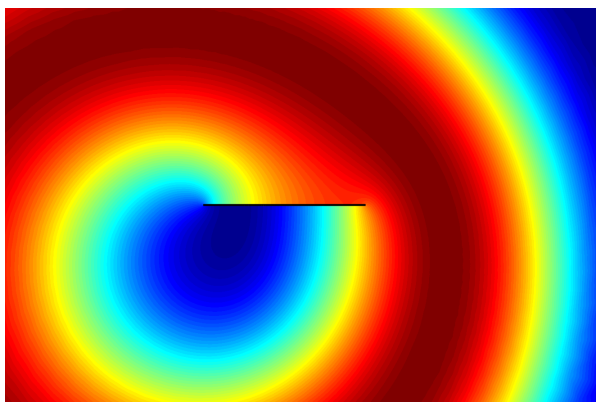

(b) $M=0$, vertical flow.

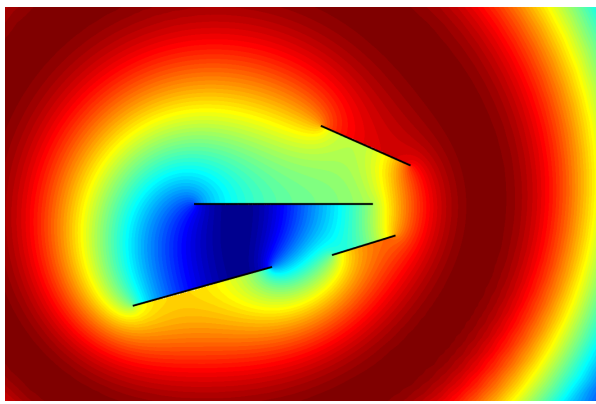

(d) $M=3$, vertical flow.

Fig. 7 The compact Green's function for an arrangement of objects in a low Mach number flow. In this case, the Mach number is 0.2. The background flow is horizontal for figures on the left hand side, and vertical for figures on the right hand side. 


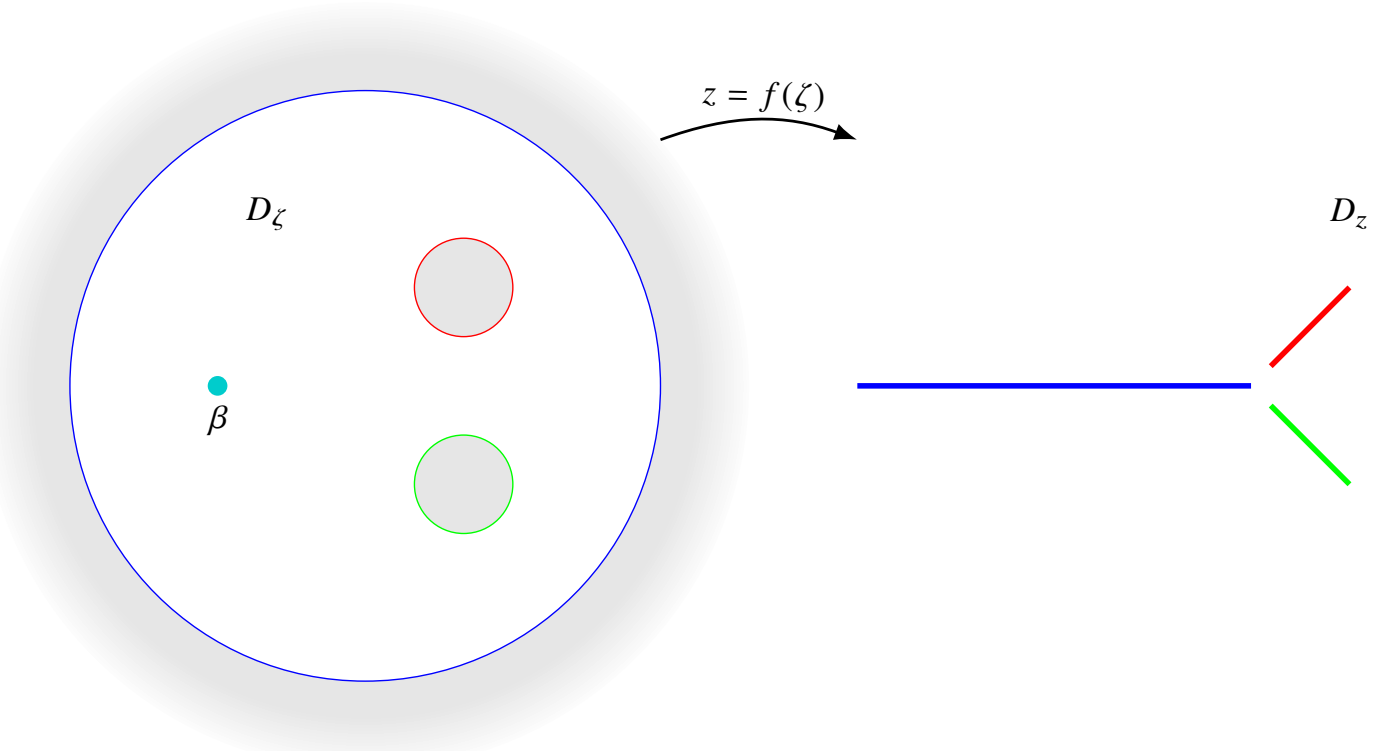

(a)

(b)

Fig. 8 The conformal mapping between a triply connected circular domain and the Kasper wing as defined in (15). The arrangement consists of the main aerofoil and two additional 'flaps' that are designed to control the vortex shedding at the trailing edge. The point $\beta$ is mapped to infinity.

In this case, analytic expressions for the conformal map are available. This conformal map is an example of a radial slit map and was derived by Nelson and Sakajo [20]. The mapping function is given by

$$
f(\zeta)=1+S \frac{\omega(\zeta, 1)^{2}}{\omega(\zeta, \beta) \omega\left(\zeta \bar{\beta}^{-1}\right)},
$$

where $S$ is some constant.

In figure 9 we plot the far-field pressure for an acoustic source near the Kasper wing for a range of wing geometries. This analysis allows us to understand the impact of the flap angle on the far-field sound. For example, the figure suggests that the blocking of sound by the flaps has a significant effect on the acoustic emissions when the flap angle is greater than $20^{\circ}$. Further work is needed to draw concrete conclusions on the sound generation for a Kasper wing.

This study could be adapted to analyse the noise generated by aerofoils in ground effect. Recent research has uncovered the form of the conformal map needed to generate the Kirchhoff vectors for any angle of attack [21]. The ensuing analysis could have implications for noise generation in take-off and landing scenarios.

\section{Conclusions and future work}

In this paper we have derived an analytic expression for the compact Green's function for an arbitrary number of objects. The principal result of the paper is to make the previously unrecognised link between the compact Green's function and the Schottky-Klein prime function. This work represents the first application of the theory of the prime function to a problem in acoustics. This link permits the calculation of the Kirchhoff vectors, which are the velocity potentials of uniform flow past the arrangement. All that remains is to calculate the conformal map from the parametric circular domain to the physical domain of interest. When the conformal mapping is not obvious, constructive tools are available [15].

In this paper we have made no attempt to satisfy the Kutta condition, or regularise the flow at any sharp edges. Whilst much research has been devoted to developing models to satisfy the Kutta condition for single bodies, there is further work to be done for scenarios involving multiple bodies. Several possible models are available [22, 24] but their application to multi-body systems requires further analysis. 


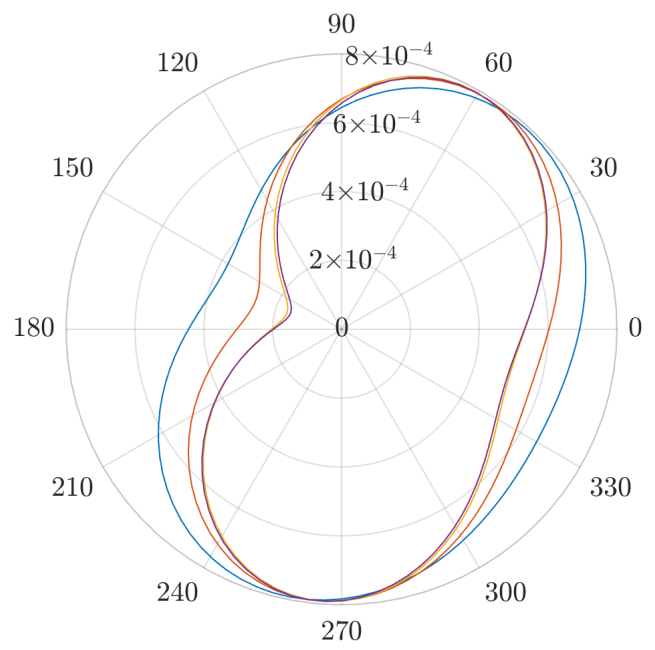

Fig. 9 The far-field pressure for an acoustic source near a Kasper wing. The colors correspond to a flap-tochord angle of $5^{\circ}, 20^{\circ}, 35^{\circ}$ and $50^{\circ}$ respectively.

The practical application of the work is broad and far reaching. Of particular interest are further investigations into multi-element high-lift aerofoils [25]. The inclusion of small amplitude pulsations is a further feature that could be incorporated into the analysis in a straightforward manner [13]. The trajectories of point vortices are already available via the Kirchhoff-Routh path function [6] so it is straightforward to apply the present theory to model vortex sound generation by multiple bodies by using the approach of Howe [1]. Essentially, many studies into the compact sound generated by a single body can now be extended to consider multiple interacting bodies.

\section{References}

[1] Howe, M. S., Theory of vortex sound, Cambridge University Press, 2003.

[2] Deglaire, P., Ågren, O., Bernhoff, H., and Leijon, M., "Conformal mapping and efficient boundary element method without boundary elements for fast vortex particle simulations," Eur. J. Mech. - B/Fluids, Vol. 27, No. 2, 2008, pp. $150-176$.

[3] Österberg, D., "Multi-Body Unsteady Aerodynamics in 2D Applied to a Vertical-Axis Wind Turbine Using a Vortex Method," Ph.D. thesis , 2010.

[4] Halsey, N. D., "Potential flow analysis of multielement airfoils using conformal mapping," AIAA J., Vol. 17, No. 12, 1979, pp. 1281-1288.

[5] Baker, H. F., Abelian functions: Abel's theorem and the allied theory of theta functions, Cambridge University Press, 1897.

[6] Crowdy, D. G., and Marshall, J., "Analytical formulae for the Kirchhoff-Routh path function in multiply connected domains," Proc. R. Soc. A Math. Phys. Eng. Sci., Vol. 461, No. 2060, 2005, pp. 2477-2501.

[7] Crowdy, D. G., and Marshall, J., "Conformal mappings between canonical multiply connected domains," Comput. Methods Funct. Theory, Vol. 6, No. 1, 2006, pp. 59-76.

[8] Crowdy, D. G., "Schwarz-Christoffel mappings to unbounded multiply connected polygonal regions," Math. Proc. Cambridge Philos. Soc., Vol. 142, No. 2, 2007, pp. 319-339.

[9] Crowdy, D. G., Surana, A., and Yick, K. Y., "The irrotational motion generated by two planar stirrers in inviscid fluid," Phys. Fluids, Vol. 19, No. 1, 2007.

[10] Crowdy, D. G., “A new calculus for two-dimensional vortex dynamics,” Theor. Comput. Fluid Dyn., Vol. 24, No. 1-4, 2010, pp. 9-24.

[11] Crowdy, D. G., "Analytical formulae for source and sink flows in multiply connected domains," Theor. Comput. Fluid Dyn., Vol. 27, No. 1-2, 2013, pp. 1-19. 
[12] Crowdy, D. G., Kropf, E. H., Green, C. C., and Nasser, M. M. S., "The Schottky-Klein prime function: A theoretical and computational tool for applications," IMA J. Appl. Math., Vol. 81, No. 3, 2016, pp. 589-628.

[13] Howe, M. S., Acoustics of fluid-structure interactions, Cambridge University Press, 1998.

[14] Crowdy, D. G., "The Schwarz-Christoffel mapping to bounded multiply connected polygonal domains," Proc. R. Soc. A Math. Phys. Eng. Sci., Vol. 461, No. 2061, 2005, pp. 2653-2678.

[15] Kropf, E., "Numerical computation of Schwarz-Christoffel transformations and slit maps for multiply connected domains," 2012.

[16] DeLillo, T. K., and Sahraei, S., "Computation of plane potential flow past multi-element airfoils using conformal mapping, revisited,” J. Comput. Appl. Math., 2018.

[17] Crowdy, D. G., "Uniform flow past a periodic array of cylinders," Eur. J. Mech. B/Fluids, Vol. 56, 2016, pp. $120-129$.

[18] Taylor, K., "A Transformation of the Acoustic Equation with Implications for Wind-Tunnel and Low-Speed Flight Tests," Proc. R. Soc. A Math. Phys. Eng. Sci., Vol. 363, 1978, pp. 271-281.

[19] Kasper, W., “Aircraft wing with vortex generation,” , jul 1974.

[20] Nelson, R., Protas, B., and Sakajo, T., "Linear feedback stabilization of point-vortex equilibria near a Kasper wing," J. Fluid Mech., Vol. 827, 2017, pp. 121-154.

[21] Baddoo, P. J., and Ayton, L. J., "Vortex equilibria in ground effect," 2018 Fluid Dyn. Conf. AIAA Aviat. Forum, American Institute of Aeronautics and Astronautics, Atlanta, Georgia, 2018, p. 2903.

[22] Howe, M. S., "Emendation of the Brown \& Michael equation, with application to sound generation by vortex motion near a half-plane," J. Fluid Mech., Vol. 329, 1996, pp. 89-101.

[23] Brown, C. E., and Michael, W. H., "Effect of leading-edge separation on the lift of a delta wing," J. Aeronaut. Sci., Vol. 21, No. 10, 1954, pp. 690-694.

[24] Saffman, P. G., Vortex Dynamics, Cambridge University Press, Cambridge, 1993.

[25] Salas, P., Fauquembergue, G., and Moreau, S., "Direct noise simulation of a canonical high lift device and comparison with an analytical model," J. Acoust. Soc. Am., Vol. 140, No. 3, 2016, pp. 2091-2100. 